\title{
Laparoscopic Management of Lately Diagnosed Triplet Heterotopic Pregnancy
}

\author{
Amal Al Mulla*, Atif B. E. Fazari, Wael A. Ismail Madkour, Alaa A. Shahin \\ Consultant OBSGYN, Dubai Fertility Centre, Dubai Health Authority, Dubai, United Arab Emirates \\ Email: *amalalmulla2020@outlook.com
}

How to cite this paper: Al Mulla, A., Fazari, A.B.E., Madkour, W.A.I. and Shahin, A.A. (2018) Laparoscopic Management of Lately Diagnosed Triplet Heterotopic Pregnancy. Open Journal of Obstetrics and $G y$ necology, 8, 51-57.

https://doi.org/10.4236/ojog.2018.81006

Received: December 10, 2017

Accepted: January 12, 2018

Published: January 15, 2018

Copyright () 2018 by authors and Scientific Research Publishing Inc. This work is licensed under the Creative Commons Attribution International License (CC BY 4.0).

http://creativecommons.org/licenses/by/4.0/

\begin{abstract}
Ruptured ectopic pregnancy is one of the outcomes of mis or delayed diagnosis and the massive hemorrhage resulting is responsible for more than three quarters of the first trimester maternal death. Simultaneous presence of intrauterine pregnancy adds obstacles for the correct diagnosis to take place. The management of this condition is a real dilemma as life threatening condition could be the only presentation which needs immediate intervention. The high index of suspicion is mandatory to ensure timely diagnosis and management to prevent stormy outcome.
\end{abstract}

\section{Keywords}

Heterotopic Pregnancy, Hemoperitoneum, Anaesthesia for Laparoscopy in Pregnancy

\section{Introduction}

Co-existence of extra as well as intra uterine pregnancy is extremely rare condition, known as Heterotopic pregnancy; the recorded incidence is $1 / 30,000$ in spontaneous pregnancies that reaches to 1:100 in cases of Assisted Reproductive treatment [1].

Reasons could be due to transfer of a large number embryos, a transfer near the uterine horn, excessive pressure on the syringe and deep insertion of the catheter during transfer, the quality of the embryos, the hormonal milieu at the moment of transfer, the use of gonadotropins, the amount of fluid used as media for the embryos [2].

We present a rare case of delayed ruptured heterotopic triplet pregnancy presented at 12 weeks gestation in compensated shock and was managed by immediate laparoscopy with favorable outcome. 


\section{Case Presentation}

A 38-year-old lady $\mathrm{P} 0+1$, conceived with Intra cytoplasmic sperm injection (ICSI) after 4 years of primary infertility. The patient diagnosed as a case of repeated implantation failure after 4 unsuccessful ICSI trials. Thrombophilia, Autoimmune and Karyotyping screening were done \& all were normal.

Three fresh euploid embryos were transferred on day 5 at blastocyst stage after preimplantation genetic diagnosis (PGD). The 3 embryos were graded according to Gardner blastocyst grading scale as 3CC. (In this scale the embryologist comment on the three components of the blastocysts (expansion, inner cell mass, trophectoderm) [3].

Human chorionic gonadotropin (hCG) hormonewas done on day 10 post embryo transfer confirmed pregnancy with a value of $234.9 \mathrm{mlU} / \mathrm{ml}$, which considered to be within the normal range for uncomplicated intrauterine pregnancy.

The first Ultra Sound Scan (USS) was at 6 weeks, revealed 2 gestational sacs, corresponds to the calculated gestational age (Figure 1).

Another USS was done at 8 weeks showed satisfactory growth for the fetuses; and the patient reported no symptoms apart from mild indigestion.

At 11 weeks, the patient arrived to the hospital in hypotensive state, recorded blood pressure was $90 / 60 \mathrm{mmHg}$, pulse rate $110 / \mathrm{min}$, oxygen saturation $99 \%$ in room air.

Generalized tenderness elicited all over the abdomen, no guarding or rigidity.

Bed side USS revealed viable intrauterine twin pregnancy and hemoperitoneum.

Hemoglobin was $6.7 \mathrm{gms} / \mathrm{dl}$. Appropriate resuscitation done and decision for emergency laparoscopy taken after discussion with the anesthetist with a presumptive diagnosis of ruptured ovarian cyst.

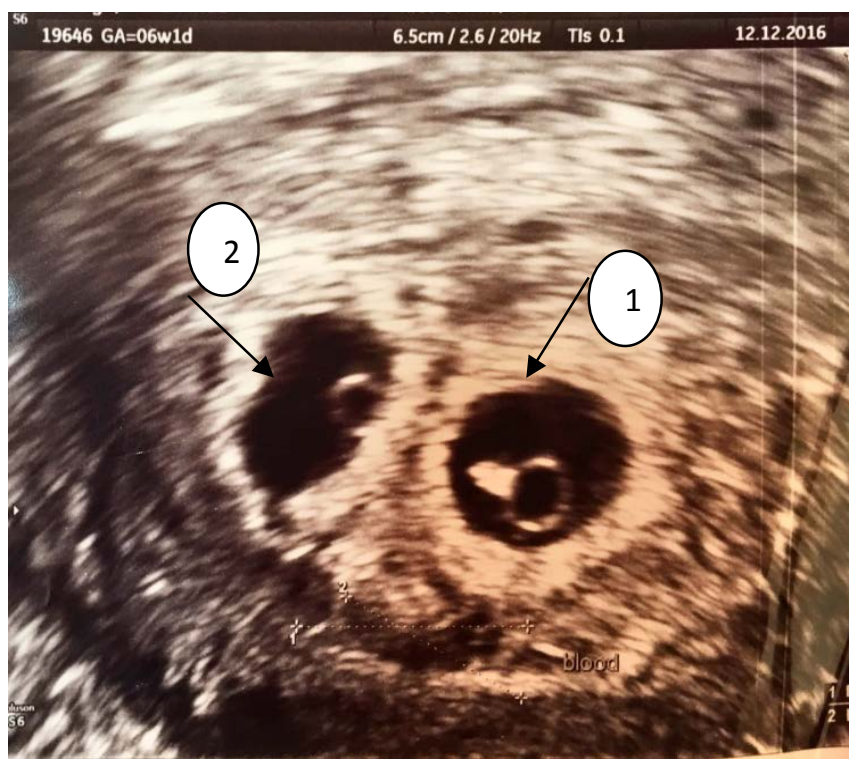

Figure 1. Ultrasound examination showed an intrauterine twin pregnancy $(1,2)$ at 6 weeks of gestations. 
Pneumoperitoneum was created using direct trocar entry at supra umbilical point to prevent injury to the gravid uterus. Two secondary ports of $5 \mathrm{~mm}$ each on each side at the level of the umbilicus were inserted.

Panoramic view of the upper abdomen anatomy was normal, approximately $800-1200 \mathrm{~mL}$ of hemoperitoneum observed, 18 weeks size gravid uterus and ruptured right fallopian tube (Figure 2).

Salpingectomy was performed, later histopathologic examination confirmed the presence of chorionic villai (Figure 3 ).

Intra operatively the patient tolerated the procedure well through appropriate transfusion of blood and blood products. Post-operative Complete blood count (CBC) were within normal values. On the next day follow up she was quite well and discharge according to the hospital protocol.

Subsquent antenatal follow up was unreamrkablewith satisfactory growth parameters for both fetuses. Patient recieved corticosteriods in view of high risk of prematurity.

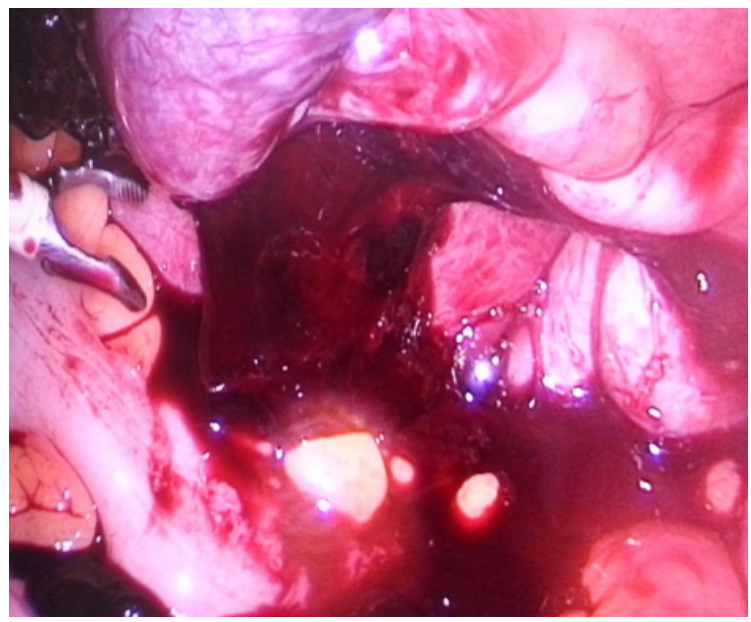

Figure 2. Haemoperitoneum demonstrated during the laparoscopy.

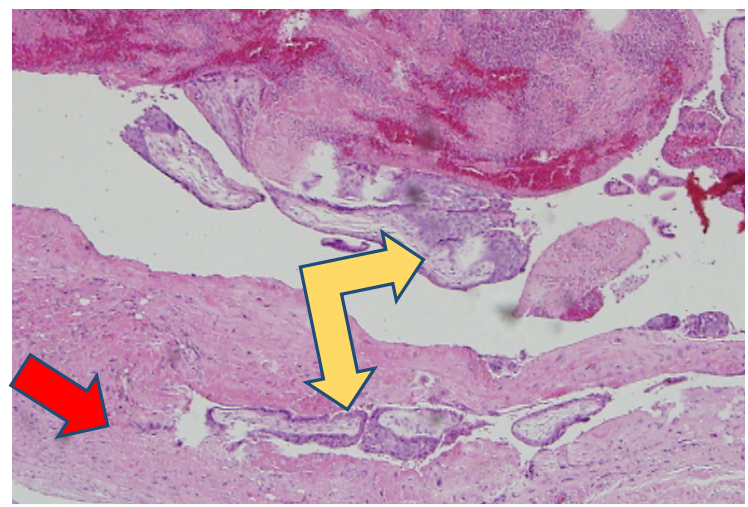

Figure 3. Portion of fallopian tube, with some chorionic villi penetrating its wall $\times 200 \mathrm{H} \& \mathrm{E}$ stain. Yellow arrow pointing to chorionic villai, red arrow pointing to fallopian tube wall. 
At 34 weeks the patient had preterm labor, with the leading twin in breach presentation, thus cesarean section was performed safely.

The dichorionic diamniotic neonates were females, with birth weights of 2460 and $2600 \mathrm{~g}$, respectively. No maternal nor neonatal complicatios. Postpartum course was unremarkable.

\section{Discussion}

Majority of reported heterotopic pregnancies are of singleton pregnancy. Triplet and quadruplet heterotopic gestation also been reported, though the incidence was extremely rare [4]. With reported survival rate of $50 \%-66 \%$ in the co-existing intrauterine pregnancy with favorable outcome.

Dr. Markus Hoopmann described a case of combined bilateral tubal and intrauterine pregnancy after IVF and embryo transfer, in 2003 that ended by missed miscarriage and conservative surgery for both tubes [5].

Salah et al., reported a case in 2011 of a combined intrauterine and intraabdominal pregnancy, diagnosed at Cesarean section at 35 weeks gestations in a 38-year-old multiparous yielded healthy twin at term [6].

Theodoros Felekis, et al. described in 2014 a case of a woman with heterotopic triplets at $9+4$ gestational week following transfer of three embryos following IVF. The ectopic tubal pregnancy was ruptured and salpingectomy was performed by laparotomy. The intrauterine pregnancy progressed to the delivery by cesarean section of two healthy twins at $36+2$ gestational age [7].

Our case presented a plethora of challenges, starting from failure to diagnose it at earlier gestation, which could be due to the fact that plasma human chorionic gonadotrophin (HCG) concentration is of no diagnostic value in multiple gestations in addition absence of symptoms added to the difficulty in diagnosis.

Subnormal hormone production by ectopic gestationsis masked by higher placental production from intrauterine pregnancy is another factor [8]. Presence of large ovaries will add difficulty to the diagnosis as the gestational sac can be missed easily.

Sonographic reports of a normal twin intrauterine pregnancy gave false reassurance. All these obstacles highlight the complexity of diagnosis.

Lack of symptoms in our patient is another factor, as reported in the literature; up to $50 \%$ of heterotopic pregnancies are asymptomatic [9].

Performing laparoscopy in the second trimester is another challenge, especially in the presence of hemoperitoneum with possible injury to the gravid uterus, or cardiovascular and respiratory alterations during the pneumoperitoneum [10].

Massive hemoperitoneum is not a contraindication for laparoscopic management ectopic pregnancy with an experienced laparoscopic surgeon [11]. However, the patient should be counselled about the possibility of converting to laparotomy if the presence of the hemoperitoneum obscures proper visualization [12]. Minimal manipulation to the gravid uterus is one of the great advantages over laparotomy. 
In our case early mobilization was our concern to guard against the occurrence of thromboembolism. This can be possible by decreasing the requirement to analgesia post operatively.

Although long-term effects of laparoscopic surgery during pregnancy on the fetus have not been well studied, but according to the literature no increase in adverse outcomes has been reported [13].

Expectant management has minimal role in handling heterotopic pregnancies. The high rupture rates with hemorrhage and shock make it imperative to treat the condition immediately upon diagnosis, to avoid maternal and fetal morbidityand mortality [14].

Giving anesthesia for pregnant lady with internal hemorrhage is the third challenge in this case, which requires well experienced anesthetist.

The associated changes with laparoscopic surgeries like decrease in the venous return and cardiac output with compensatory tachycardia is aggravated by the presence of internal hemorrhagein addition to hypoxemia resulted from decrease in the functional residual capacity due to compression by the elevated diaphragm. Maintaining the abdominal pressure around $12 \mathrm{mmHg}$ is helpful.

Close monitoring through invasive arterial blood pressure is mandatory which allows vital signs monitoring as well as frequent blood gas samples, thus optimum guidance guaranteed for fluid, blood and blood products replacement to maintain acid base balance.

Managing the ventilator parameters through controlled ventilation is fundamental for adequate oxygen and carbon dioxide levels aiming for adequate oxygenation for the mother and the growing fetuses. Many studies support that a positive end-expiratory pressure (PEEP) of $5 \mathrm{~cm} \mathrm{H2O}$ is ideal to minimize lung atelectasis and to prevent hypoxia [15].

\section{Conclusions}

Despite the vast progress in medical knowledge and the use of advanced and sophisticated improved reproductive technologies, heterotopic pregnancy still remains a diagnostic and therapeutic challenge to practitioners.

The presence of an intrauterine pregnancy in an asymptomatic woman should not exclude the diagnosis of a concurrent extra uterine pregnancy. Thus, it is good practice to advocate a thorough evaluation of the adnexa in all pregnancies especially for those cases who conceived with Assisted Reproductive Techniques (ART). Possibility of heterotopic pregnancy in ART conception should be one of the probable diagnoses in women who present with acute abdomen even in the second trimester.

Laparoscopy should be offered for all patients, and hemoperitoneum should not prevent this service if experienced personnels are available.

Limiting the number of embryos transferred, according to the guidelines established by international committees for assisted reproductive technology, could be beneficial. 


\section{Consent}

Informed consent was obtained from the patient for publication of this case report and any accompanying images.

\section{Conflict of Interests}

The authors declare that they have no conflict of interests regarding the publication of this paper.

\section{References}

[1] Spandana, J.C., Kanakannavar, S.S., Umashankar, K.M. and Manuja, N. (2017) Spontaneous Heterotopic Pregnancy with Tubal Rupture. International Journal of Reproduction, Contraception, Obstetrics and Gynecology, 6, 5148. https://doi.org/10.18203/2320-1770.ijrcog20175042

[2] Divry, V., Hadj, S., Bordes, A., Genod, A. and Salle, B. (2007) Case of Progressive Intrauterine Twin Pregnancy after Surgical Treatment of Cornual Pregnancy. Fertility and Sterility, 87, 190.e1-190.e3. https://doi.org/10.1016/j.fertnstert.2006.04.053

[3] Balaban, B. and Gardner, D.K. (2013) Morphological Assessment of Blastocyst Stage Embryos: Types of Grading Systems and Their Reported Outcomes. Human Gametes and Preimplantation Embryo, Chapter4, 31-43.

[4] Noor, N., Bano, I. and Parveen, S. (2012) Heterotopic Pregnancy with Successful Pregnancy Outcome. Journal of Human Reproductive Sciences, 5, 213-214. https://doi.org/10.4103/0974-1208.101024

[5] Hoopmann, M. (2003) Case Report: Heterotopic Triplet Pregnancy with Bilateral Tubal and Intrauterine Pregnancy after IVF. Reproductive BioMedicine, 6, 345-348. https://doi.org/10.1016/S1472-6483(10)61855-X

[6] Ahmed, S.R., Abdelhafez, H.A., Ibrahim, K.M. and Alsammani, M.A. (2013) Heterotopic Pregnancy Following IVF Carried to Term Pregnancy: A Case Report. IJCRR, 5, 93-96.

[7] Felekis, T., Akrivis, C., Tsirkas, P. and Korkontzelos, I. (2014) Heterotopic Triplet Pregnancy after In Vitro Fertilization with Favorable Outcome of the Intrauterine Twin Pregnancy Subsequent to Surgical Treatment of the Tubal Pregnancy. Case Reports in Obstetrics and Gynecology, 2014, Article ID: 356131. https://doi.org/10.1155/2014/356131

[8] Tummon, I.S., Whitmore, N.A., Daniel, S.A., Nisker, J.A. and Yuzpe, A.A. (1994) Transferring More Embryos Increases Risk of Heterotopic Pregnancy. Fertility and Sterility, 61, 1065-1067.

[9] Sun, S.Y., Araujo Jr., E., Elito Jr., J., et al. (2012) Diagnosis of Heterotopic Pregnancy Using Ultrasound and Magnetic Resonance Imaging in the First Trimester of Pregnancy: A Case Report. Case Reports in Radiology, 2012, Article ID: 317592. https://doi.org/10.1155/2012/317592

[10] Hasson, J., Tsafrir, Z., Azem, F., et al. (2010) Comparison of Adnexal Torsion between Pregnant and Nonpregnant Women. American Journal of Obstetrics \& Gynecology, 202, 536.e1-6. https://doi.org/10.1016/j.ajog.2009.11.028

[11] Chaudhary, P., Manchanda, R. and Patil, V.N. (2013) Retrosepective Study on Laparoscopic Management of Ectopic Pregnancy. The Journal of Obstetrics and Gynecology of India, 3, 173-176. 
[12] Jazayeri, A. and Rivlin, M.E. (2015) Surgical Management of Ectopic Pregnancy Updated. Feb. 16. https://emedicine.medscape.com/article/267384-overview

[13] Bugatto, F., Quintero-Prado, R., Kirk-Grohar, J., Melero-Jiménez, V., HervíasVivancos, B. and Bartha, J.L. (2010) Heterotopic Triplets: Tubal Ectopic and Twin Intrauterine Pregnancy. A Review of Obstetric Outcomes with a Case Report. Archives of Gynecology and Obstetrics, 282, 601-606.

[14] Nathan, R. and Joseph, G. (1996) Review: Heterotopic Pregnancy and Assisted Reproduction: An Update. Journal of Assisted Reproduction and Genetics, 13, 594-601.

[15] Arati, S. and Ashutosh, N. (2010) Secrets of Safe Laparoscopic Surgery: Anaesthetic and Surgical Considerations. Journal of Minimal Access Surgery, 6, 91-94. 